\title{
An improved probabilistic method for screening safety-related human actions in nuclear power plants
}

\author{
Li Zhang, ${ }^{\mathrm{a}, \mathrm{b}}$, Chunbo Wang ${ }^{\mathrm{b}}$, Enrico Zio ${ }^{\mathrm{c}, \mathrm{d}}$, Yanhua Zou ${ }^{\mathrm{a}, *}$, Jianjun Jiang ${ }^{\mathrm{a}}$, Yanzi Liu ${ }^{\mathrm{e}}$, \\ Zhaopeng Liu ${ }^{\mathrm{e}}$ \\ a Institute of Human Factors Engineering \& Safety Management, Hunan Institute of Technology, Hengyang, Hunan 421002, China \\ ${ }^{\mathrm{b}}$ School of Nuclear Science and Technology, University of South China, Hengyang, Hunan 421001, China \\ ${ }^{\mathrm{c}}$ Dipartimento di Energia, Politecnico di Milano, Milano 20156, Italy \\ ${ }^{\mathrm{d}}$ Chair System Science and the Energy Challenge, Fondation Electricité de France (EDF), CentraleSupélec, Université Paris Saclay, Chatenay-Malabry 92290, France \\ e State Key Laboratory of Nuclear Power Safety Monitoring Technology and Equipment, China Nuclear Power Design Company LTD., Shenzhen, Guangdong 518045, \\ China
}

\section{A R T I C L E I N F O}

\section{Keywords:}

Nuclear power plant (NPP)

Human actions

Probabilistic screening method

Human action risk achievement worth (HRAW)

Human factors engineering (HFE)

\begin{abstract}
A B S T R A C T
For the reliability and safety of nuclear power plants (NPPs), their design and operation must follow the relevant principles of human factors engineering (HFE). As various human actions are involved in the operation of NPPs, there is a need to screen human actions first and, then, focus the analysis on the critical ones. In the past, different NPPs have adopted different standards in screening human actions and many of these standards lack theoretical support. In this study, the concept of human action risk achievement worth (HRAW) is introduced to support probabilistic screening. A new probabilistic method for screening safety-related human actions in NPPs is proposed. The results of a case study indicate that the proposed method performs well and better than the previous probabilistic screening method typically used, it also implies that this method can provides methodological support to the probabilistic screening method of human actions. On the one hand, utilizing this method to screen human actions can help identifying those actions that are critical for the safety of NPPs. On the other hand, it can reduce the workload and improve work efficiency. The method is traceable and easy to use. It not only can be used in the design of NPPs, but also can provide guidelines for reviewers to evaluate the NPPs safety.
\end{abstract}

\section{Introduction}

After the accidents at the Three Mile Island and Chernobyl nuclear power plants (NPPs), the influence of human errors on NPPs has aroused wide concern (Dhillon, 2013; Liu and Li, 2014). For this reason, Human Factors Engineering (HFE) has become important in the design and operation of NPPs (Zhang, 2002). In the design of NPPs, the twelve elements of HFE must be taken into consideration, particularly the human reliability analysis (HRA) (Ming et al., 2017; Zou et al., 2017). In 2012, the U.S. Nuclear Regulatory Commission (NRC) amended the section of HRA in the Human Factors Engineering Program Review Model (NUREG-0711), Version 3, and renamed it as Treatment of Important Human Actions, which emphasizes the detailed analysis of human actions to reduce human errors (O'Hara et al.,2012).

In the past, during the design of NPPs, analysis of human actions usually adopted the qualitative analysis of HRA. As an essential part of the HFE review, human actions have drawn the attention again after NUREG-0711 upgrade. For example, the Office for Nuclear Regulation (ONR) proposed that detailed explanations on the contribution of human actions to system risk should be provided (ONR, 2014); the National Nuclear Safety Administration of China (NNSA) also put forward the requirement of detailed analysis of human actions during project review. Considering the numerosity and variety human actions in the operation of NPPs, it is necessary to screen for the critical human actions and identify the corresponding risk contributions.

The present ways aim at reducing the workload of designers by

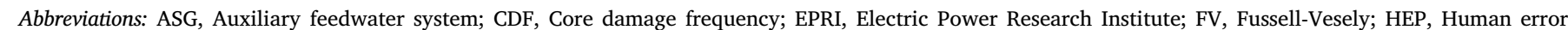

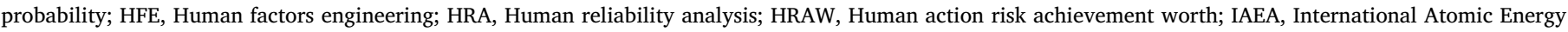

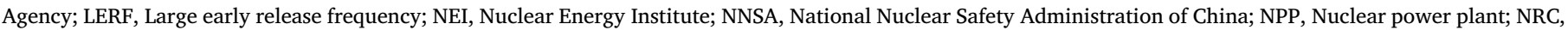

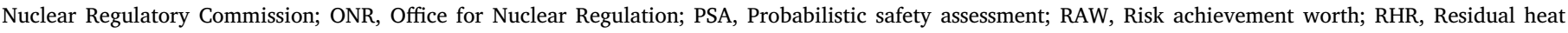
removal system; RRW, Risk reduction worth; SDP, Significance determination process; SGTR, Steam generator tube rupture

* Corresponding author.

E-mail address: zouyanhua@163.com (Y. Zou). 
providing a theoretical basis for human-system interface (HSI) design, procedure development and improvement of training program. The screening of human actions adopts the concept of importance measures used to rank components based on their contributions to system risk, within a probabilistic safety assessment (PSA) framework. Importance measures are often used in the selection of critical components, for reliability design and maintenance optimization, etc. (Sallak et al., 2013; Zio and Luca, 2003). Typical importance measures include risk achievement worth (RAW), Fussell-Vesely (FV) and risk reduction worth (RRW) (Vesely et al., 1994; Higgins et al., 2007; Mancuso et al., 2017; Zio, 2007). For a probabilistic method to screen human actions, it is important to use the appropriate value of importance measure as the risk boundary, so as to ensure that the screening results are conservative. In previous studies, the risk boundary selected for screening human actions was usually set the same as the value used for the selection of critical components. For example, the Nuclear Energy Institute (NEI) and the Electric Power Research Institute (EPRI) adopt 2 as the risk boundary of the screening of RAW and 0.005 is considered as the risk boundary of the screening of FV (NEI, 2005; True et.al., 1995). This standard had also been recognized by the International Atomic Energy Agency (IAEA) (IAEA, 2001). However, there are significant differences between human actions and components functions, so it may not be appropriate to apply the standard for components directly to the screening of human actions. For example, operators are different from components, and when a certain threshold is exceeded, the component will be unavailable, but the operator still has the possibility to successfully perform the actions. For this reason, Westinghouse made adjustments to the value of risk boundary when screening human actions for the design of AP600 and AP1000 units. For the baseline PSA, 3 is set as the risk boundary of the RAW and 0.1 is set as the risk boundary of the RRW; for the focused PSA, 2 is set as the risk boundary of the RAW and 0.05 is set as the risk boundary of the RRW (Kerch et al., 1997; Schulz et al., 2006). Unfortunately, Westinghouse did not provide the scientific basis for the selection of the values. Higgins et al. (2002) have established a screening method of human actions by using the significance determination process (SDP). But this method has not been verified in practice, and still remains in the theoretical research stage.

In this paper, we introduced the human action risk achievement worth (HRAW) to support the probabilistic screening method for safetyrelated human actions. A case study indicates that this method performs well and better than the previous probabilistic screening method typically used. On the one hand, the adoption of this method can provide methodological support for the probabilistic screening of human actions. On the other hand, it can also provide the basis for the design of NPPs.

The rest of this paper is structured as follows. In Section 2, we recall the PSA important measures and, then, propose the approach for HRAW determination and the probabilistic screening method. In Section 3, we present a case study related to a NPP under construction in China. The screening results based on the HRAW and FV are presented and compared with the results of the commonly used probabilistic screening method. In Section 4, we discuss the main results and the significance of the method proposed in this paper.

\section{Probabilistic method for screening safety-related human actions}

\subsection{PSA importance measures}

Core damage frequency (CDF) and large early release frequency (LERF) are the main indicators used for evaluating the design safety of NPPs (Siu et al., 2016). Both CDF and LERF are obtained through a PSA. To obtain these values, the accident sequences from initial events must be defined and, then, their consequences and frequencies of occurrences must be quantified on the basis of the event frequency and compared at human reliability data. CDF is a value obtained from Level 1 PSA, while
LERF is a value obtained from Level 2 PSA (Zhu, 2004; Chen et al, 2013). CDF and LERF have similar meaning within the probabilistic method for screening human actions, for ease of discussion, in this study we refer only to CDF.

Importance measures are often used in PSA for the safety analysis of NPPs. Different importance measures have different definitions and meanings (Borst and Schoonakker, 2001). RAW represents the contribution of a component to avoiding system failure. For this reason, some authors refer to RAW as a safety importance measure (Prasad et al., 2014). It is defined as follows:

$\operatorname{RAW}(\mathrm{i})=\frac{\mathrm{R}_{\mathrm{i}+}}{\mathrm{CDF}}$

where $R_{i+}$ is the value of CDF when the probability of component $i$ failure is 1 .

FV (Rebaiaia et al., 2015) and RRW (Prasad et al., 2014) represent the system risk contributions of the failure of the component, so they are also called risk importance measures. They are defined as follows:

$\mathrm{RRW}(\mathrm{i})=\frac{\mathrm{CDF}}{\mathrm{R}_{\mathrm{i}-}}$

$\mathrm{FV}(\mathrm{i})=\frac{\mathrm{R}_{\mathrm{i}}}{\mathrm{CDF}}$

where $R_{i-}$ is the CDF contributed by the cut sets excluding the component $\mathrm{i}$; $\mathrm{R}_{\mathrm{i}}$ is the CDF contributed by the minimal cut sets including the component $\mathrm{i}$.

CDF and $R_{i+}$ can be expressed in terms of $R_{i-}$ and $R_{i}$, as:

$\mathrm{CDF}=\mathrm{R}_{\mathrm{i}}+\mathrm{R}_{\mathrm{i}-}$

$\mathrm{R}_{\mathrm{i}+}=\mathrm{R}_{\mathrm{i}}(\mathrm{Pi}=1)+\mathrm{R}_{\mathrm{i}-}$

Then, there is a mathematical relationship between RRW and FV, i.e., $F V=1-1 / R R W$ (Kim et al., 2017). In view of their wide application in NPPs safety analysis, in this paper we look at FV and RAW for screening human actions.

In the calculation of RAW, $R_{i+}$ is based on the assumption that the failure probability of component $i$ is 1 . The designers can consider this assumption in the design of NPPs, because during the life cycle of NPPs, components may indeed be unavailable due to maintenance or failure. But for human actions, assuming that error probability of performing actions is 1 is at least arguable, as consideration should be given the operators' physiological limits, for performing actions. Then, for the screening of human actions, FV is applicable whereas RAW is inapplicable and we put forward the concept of HRAW for human actions screening. HRAW is defined as the increase in the risk to the system caused by humans in the performance of an action i under extreme conditions. Under extreme conditions, the human error probability (HEP) can be high but it is inappropriate to assume that it is 1 , because of the operators' stress characteristics. So, the definition of HRAW is as follows:

$\operatorname{HRAW}(\mathrm{i})=\lim _{\mathrm{p}(\mathrm{i}) \rightarrow 1} \frac{\mathrm{R}_{\mathrm{i}}+\mathrm{R}_{\mathrm{i}-}}{\mathrm{CDF}}$

Considering the definition of $\mathrm{R}_{\mathrm{i}}$ and $\mathrm{R}_{\mathrm{i}-\text {, }}$, Eq. (6) can be rewritten as Eq. (7).

$\operatorname{HRAW}(\mathrm{i})=\lim _{p(i) \rightarrow 1} \frac{R_{i}+R_{i-}}{C D F}=\frac{R_{i-}+\lim _{p i \rightarrow 1} R_{i}}{C D F}$

By comparing Eqs. (5) and (7), we can establish a relationship between HRAW and RAW for human action i, in terms of an infinitesimal $o(\alpha)$ :

$\operatorname{HRAW}(\mathrm{i})=\frac{\mathrm{R}_{i+}}{\mathrm{CDF}}-o(\alpha)=\mathrm{RAW}(\mathrm{i})-o(\alpha)$

$o(\alpha)$ represent the possibility of operator successfully perform action i under extreme conditions. 


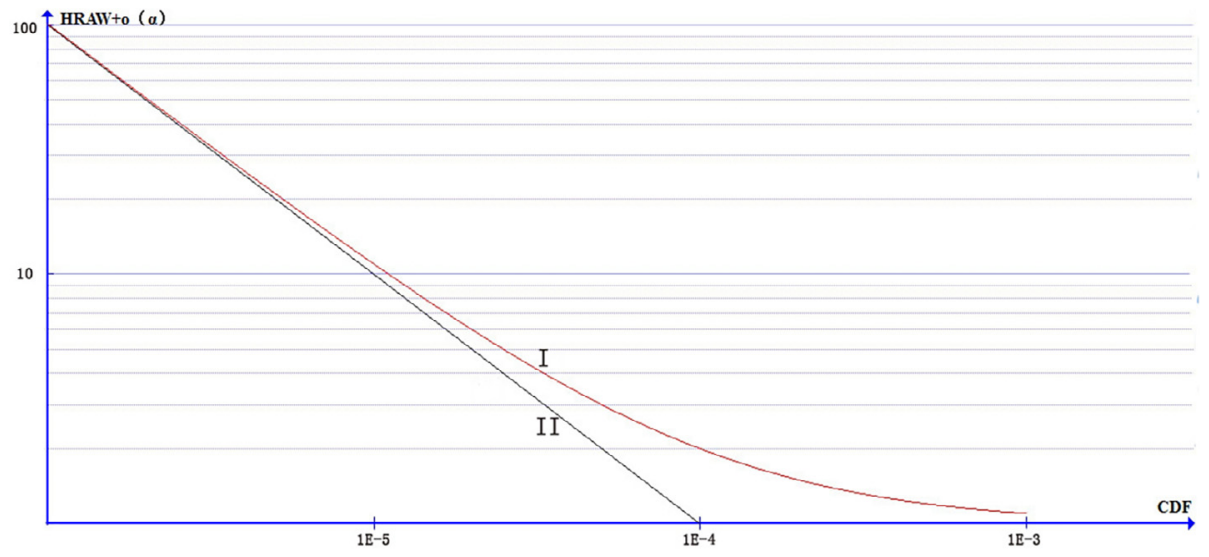

Fig. 1. Probabilistic screening of human actions.

\subsection{Determination of HRAW}

From Eq. (8), we have:

HRAW $(\mathrm{i})=\frac{\mathrm{R}_{\mathrm{i}+}}{\mathrm{CDF}}-o(\alpha)=\frac{\mathrm{R}_{\mathrm{i}+}-\mathrm{CDF}+\mathrm{CDF}}{\mathrm{CDF}}-o(\alpha)=\frac{\Delta \mathrm{R}}{\mathrm{CDF}}+1-o(\alpha)$

where $\triangle \mathrm{R}=\mathrm{R}_{\mathrm{i}+}$-CDF.

Assigning the value of probabilistic safety goal $(1 E-4)$ to the $R_{i+}$ and $\triangle R$ in the Eqs. (8) and (9) respectively, referring to some regulations and regulatory documents, (NNSA, 2006; IAEA, 2012; U.S. NRC, 2011), we have:

HRAW $+o(\alpha)=\frac{10^{-4}}{\mathrm{CDF}}$

HRAW $+o(\alpha)=\frac{10^{-4}}{\mathrm{CDF}}+1$

Fig. 1 shows these relations in the logarithmic coordinate system. Curve I corresponds to Eq. (10) and curve II corresponds to Eq. (11), and the human actions can be mapped into the points in Fig. 1. For CDF $<1 \mathrm{E}-5$, the curves I and II are basically coincident; when $1 \mathrm{E}-5<\mathrm{CDF}<1 \mathrm{E}-4$, there is a significant difference between the two curves. We do not consider CDF $>1 \mathrm{E}-4$, it does not satisfy the requirement of the probabilistic safety goal.

The points above curve I represent the fact that when their corresponding actions are performed under extreme conditions, the increased risk of the NPPs is greater than the probabilistic safety goal. Also the points above curve II represent the fact that when their corresponding actions are performed under extreme conditions, the CDF of the NPPs is greater than the probabilistic safety goal. So, for conservation the points above curve II should be screened out for serious consideration.

As the probabilistic method for screening human actions is based on the results of PSA, it is also affected by the uncertainties of the PSA. There are two types of uncertainties typically considered in PSA: one is uncertainty caused by the stochasticity of some physical events, and the other is cognitive uncertainty related to the availability of human cognition (Zheng and Zhou, 2011). To account for this, a certain safety margin should be reserved, based on the probabilistic safety goal. For this, we assign a new value to $\triangle R$, smaller by an order of magnitude than the probabilistic safety goal:

HRAW $+o(\alpha)=\frac{10^{-5}}{\mathrm{CDF}}+1$

The curve III in the Fig. 2 corresponds to the Eq. (12). Curve III covers all the regions of curve II including the uncertainty, thus reserving a margin for the conservation of the screening. Curve III is then taken as the risk boundary and the human actions represented by the points above curve III are considered safety-critical. We can further divide the Fig. 2 into three parts: the area above curve II is the high-risk area, the middle area between curves II and III is the medium-risk area, the area below curve III is the low-risk area.

For general practical use, we notice that the values of $R_{i+}$ and $\triangle R$ can be changed according to the different probabilistic safety goals performed by different NPPs. $R_{i+}$ in Eq. (8) should be assigned the value of probabilistic safety goal, and $\triangle R$ in Eq. (9) should be assigned a value smaller by an order of magnitude than the probabilistic safety goal. Also, the risk boundary value of HRAW should be an integer, to avoid misunderstanding, because this value is based on estimated results from a PSA rather than deterministic calculations.

\subsection{Probabilistic screening method}

In order to ensure the completeness of the screening results, in addition to utilizing the HRAW for screening human actions, it is necessary to perform the screening again by using the FV. When we utilizing the $\mathrm{FV}$, the value of risk boundary adopts the value used in the selection of critical components, $\mathrm{FV}<0.005$ represents the human action is low risk; $0.005<\mathrm{FV}<0.1$ represents the human action is medium risk; $\mathrm{FV}>0.1$ represents the human action is high risk.

After conducting the human actions screening by using the HRAW and FV respectively, we need to make a comprehensive assessment to determine which risk area the actions belong to. For the same human action, if the results screened by the HRAW and FV are different, this action should be classified into the area with higher risk. For example, if a human action was identified as medium risk based on the value of HRAW, while it was identified as high risk based on the value of FV, then finally this human action should be identified as high risk. For eliminating the uncertainty caused by PSA, the screening results need to be submitted to the experts for review. Fig. 3 is the framework of probabilistic screening method.

The dependency among human actions is an important factor in the analysis (Čepin, 2008), and this part of work has been considered in the preliminary safety analysis report. This paper emphasize on the screening of safety-related human actions based on the results of preliminary safety analysis report. So, we do not have specific relevant contents about dependency among human actions in this paper. In this work, there are two possible source of uncertainty. One is from truncation limit in probabilistic safety assessment, the other is the appropriateness of the value of $\triangle R$ and $R_{i+}$ we used in the paper (Apostolakis, 1989; Čepin, 2005). The value of uncertainty will be analyzed in the further work. 


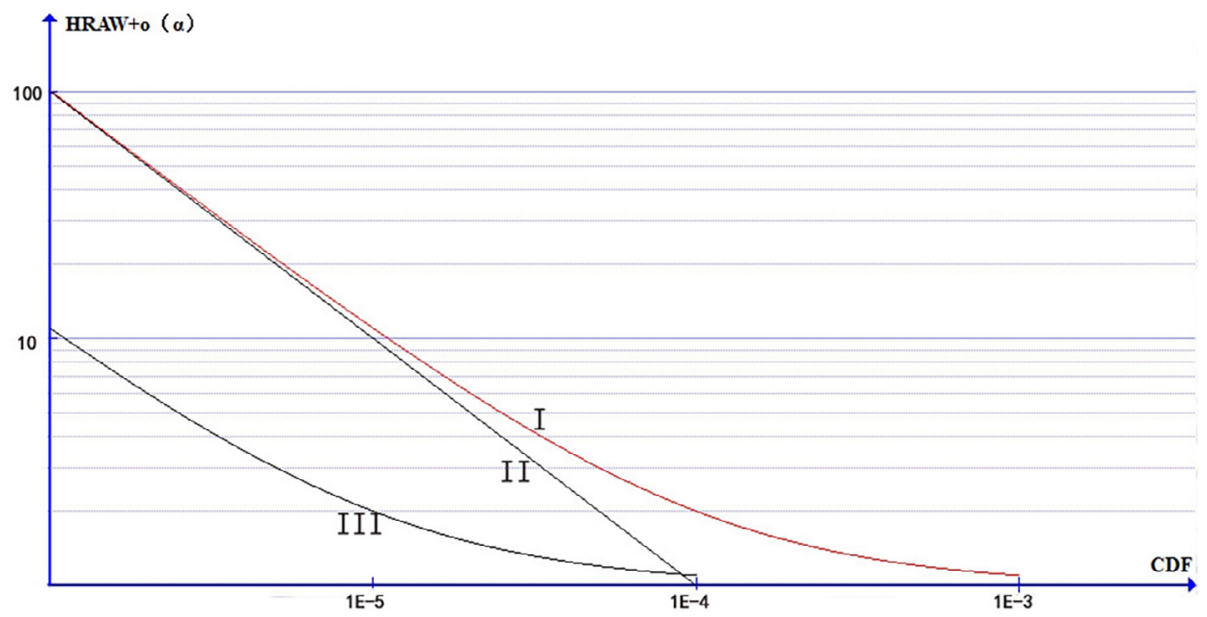

Fig. 2. Risk regionalization.

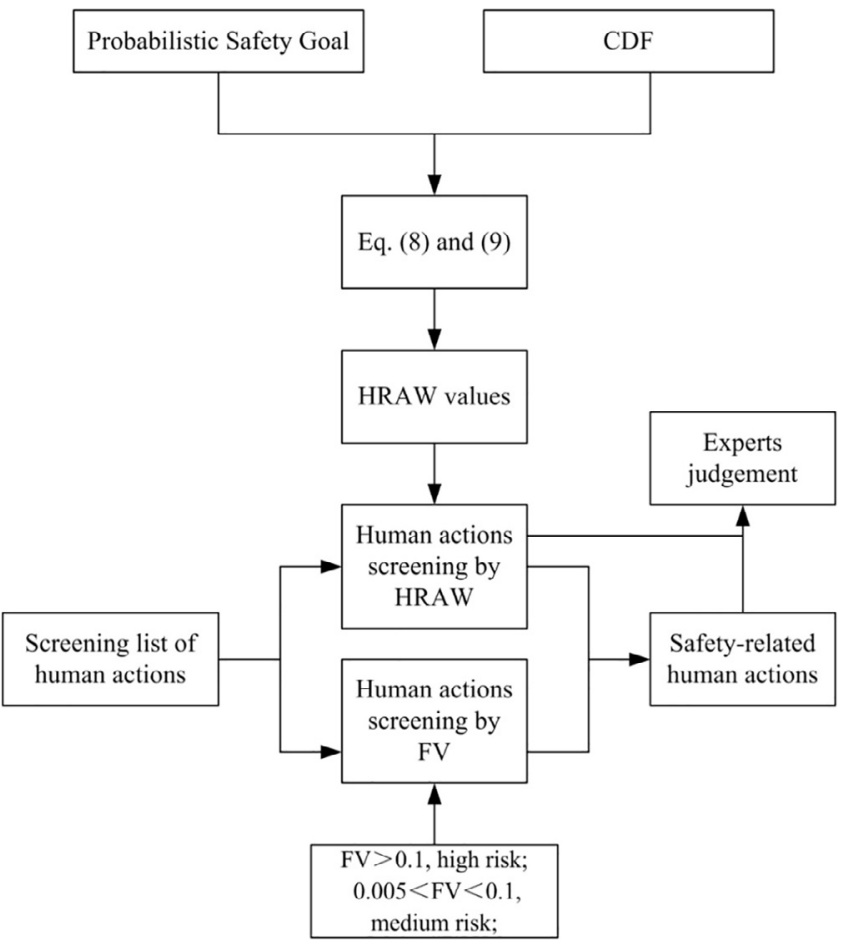

Fig. 3. Framework of probabilistic screening method.

\section{Case study}

\subsection{Background}

The PSA for a NPP under construction in China has been conducted and the preliminary safety analysis report has been submitted to the Nuclear Safety Center (NSC). In light of the design and construction process of NPPs, the screening and analysis of human actions are required at this stage. We take this NPP as an example, using the probabilistic screening method to analyze the human actions. The preliminary safety analysis report gives a CDF for this NPP equal to 4.78E -7 per reactor year. As required by Chinese regulations HAD102/17 "Assessment and Validation on the Power and Safety of Nuclear Power Plant", the probabilistic safety goal is CDF $<1 \mathrm{E}-5$ per reactor year for existing NPPs and the CDF of the probabilistic safety goal for new NPPs should be $1 \mathrm{E}-5$ per reactor year.

Then, for Eq. (8), we set $R_{i+}$ as $1 E-5$ and $C D F$ as $4.78 E-7$, and then we have the Eq. (13) below; for Eq. (9), we set $\triangle R$ as $1 E-6$, and then we have the Eq. (14) below:

$$
\begin{aligned}
& \text { HRAW }+o(\alpha)=\frac{10^{-5}}{4.78 \times 10^{-7}} \approx 20 \\
& \text { HRAW }+o(\alpha)=\frac{10^{-6}}{4.78 \times 10^{-7}}+1 \approx 3
\end{aligned}
$$

Based on this calculation, the value of 20 separates medium risk from high risk, and the value of 3 separates medium risk and low risk, seen in Fig. 4.

\subsection{Screening results}

Tables 1 and 2 provide the results of screening by the HRAW and FV respectively for a number of human actions in the NPP under construction.

Among the 11 human actions sorted out by the probabilistic screening method, "operation possible-fixed-line operation" is an operation of high risk and the other 10 actions are of medium risk. Human actions of low risk are not reported in Tables 1 and 2, because of little danger to the NPPs safety. The "Manual connection of other ASG water tanks fails" appears many times in Tables 1 and 2 with different values, the reason for this is that the error probability in operations to different ASG water tanks is different, so the values of the importance measures are correspondingly different.

\subsection{Comparative analysis}

As discussed in Section 1, in the NPPs engineering practice, the screening of human actions generally adopts the standard for selection of the critical components. This standard defines that if the values of RAW is larger than 2 or the FV is larger than 0.005 , the corresponding human actions are safety-related. Based on this standard, there are two extra human actions compared with Tables 1 and 2: one is cooling and depressurization of the primary system to achieve the conditions for starting the residual heat removal (RHR) system; the other is starting the RHR system itself. The following is the qualitative analysis for these two human actions.

(1) For the first one. Steam Generator Tube Rupture (SGTR) is one of the design basis accidents of the NPPs. When analyzing this accident, it was assumed that the NPPs system deals with the accident automatically in the first $30 \mathrm{~min}$. After, the operators take over the system. The main tasks of the operator are to identify and isolate the damaged steam generator first, and then cool and depressurize 


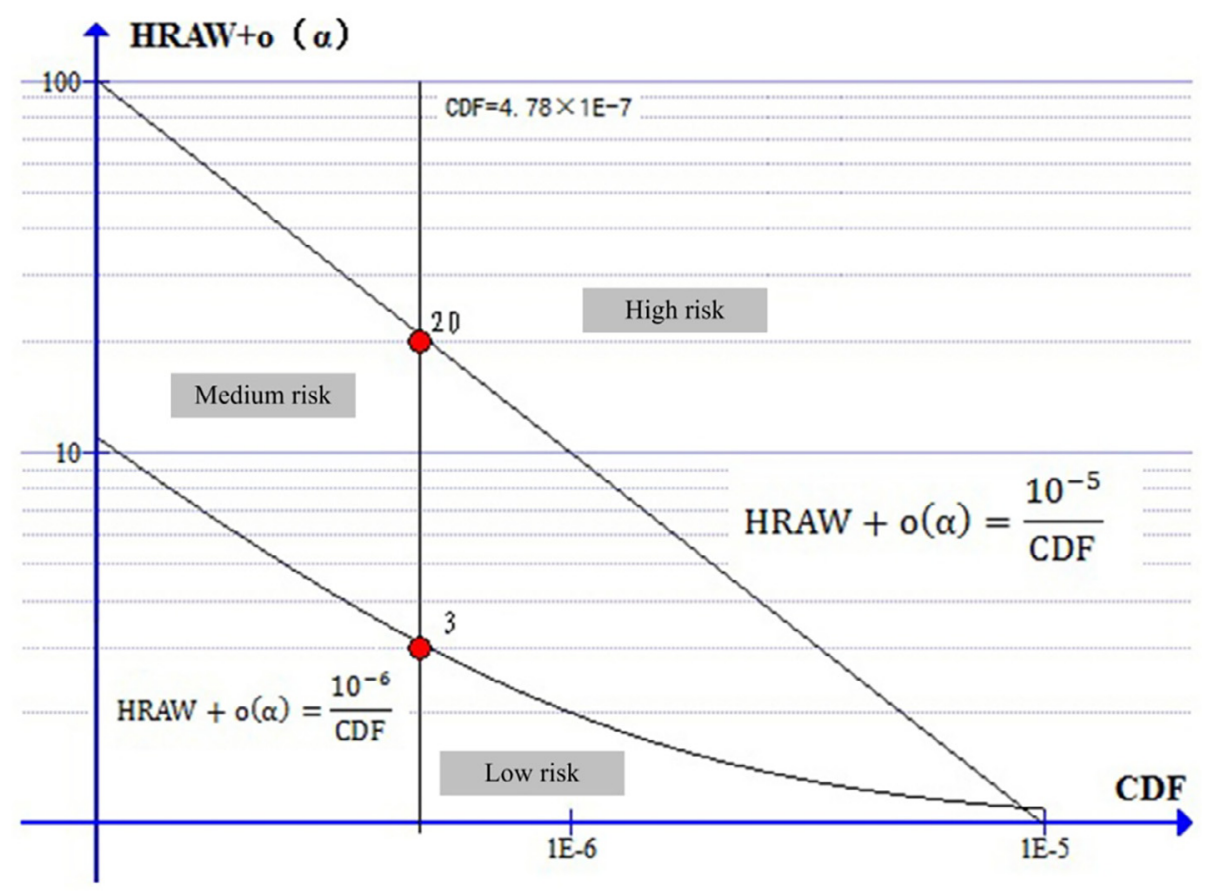

Fig. 4. Risk regionalization.

Table 1

Results of Screening by HRAW.

\begin{tabular}{lll}
\hline Description & HRAW & Explanation \\
\hline $\begin{array}{l}\text { Manual isolation of dilution source or corrective } \\
\quad \text { action operation fails - EBS is done }\end{array}$ & $1.52 \mathrm{E}+01$ & Medium risk \\
$\begin{array}{l}\text { Operator fails to switch to CHR system manually } \\
\text { Operation possible-fixed-line operation fails-1 }\end{array}$ & $1.29 \mathrm{E}+01$ & Medium risk \\
Restarting secondary circuit side cooling fails in a & 7.06E + 01 & Medium risk \\
$\quad$ shutdown condition & & Medium risk \\
Manual start of SBO diesel engine fails & $5.66 \mathrm{E}+00$ & Medium risk \\
Manual connection of other ASG-2 water tanks fails & $4.22 \mathrm{E}+00$ & Medium risk \\
Manual connection of other ASG-3 water tanks fails & $4.01 \mathrm{E}+00$ & Medium risk \\
Manual start of low head injection system-under & $3.72 \mathrm{E}+00$ & Medium risk \\
$\quad$ RHR mode & & \\
Manual connection of other ASG-1 water tanks fails & $3.51 \mathrm{E}+00$ & Medium risk \\
Operation possible-fixed-line operation fails-2 & $3.03 \mathrm{E}+00$ & Medium risk \\
\hline
\end{tabular}

*EBS: Emergency Boration System; CHR: Containment Heat Removal. SBO: Station Black Out; ASG: Auxiliary Feedwater System; RHR: Residual Heat Removal System.

the primary system to achieve the conditions for starting the RHR system; finally, to make reactor safe shutdown. If we adopt the current practice screening methods for analyzing this accident, this action is classified as a safety-related human action. But this is unreasonable because this action is performed later in the SGTR accident and the NPP is controllable at that time, the operators' stress level is low and they have enough time to complete this action with low error probability. So, this action should not be identified as safety-related.

(2) For the second one. The RHR system is generally put into use in the second phase of the cold shutdown, to exhaust the heat generated by the remaining power. Whether the reactor is shutdown under normal operation or during an accident, the RHR system is started at the final stage when the reactor power is low and the operator has enough time to perform the necessary actions. Also in this situation the operators can be considered to have low psychological load and stress load. Thus, this human action should not be classified as safety-related.

Finally, we note that according to the original method, the human actions are classified only either as safety-related or non safety-related. Differently, in our proposed method, we classify the human actions into three classes: low risk, medium risk and high risk. The high-risk actions threaten the safe operation of NPPs if there is any error in these actions, it will lead the CDF to values higher than the probabilistic safety goal. Therefore, analyzing the human actions with high-risk can lead the designers to focus on these actions from the very beginning.

\section{Discussion and conclusion}

The screening of human actions should be conducted at the early

Table 2

Results of Screening by FV.

\begin{tabular}{|c|c|c|}
\hline Description & FV & Explanation \\
\hline Operation possible-fixed-line operation fails- 2 & $2.21 \mathrm{E}-01$ & Medium risk, then raise to high risk \\
\hline Manual low-pressure cooling at full speed fails & $3.34 \mathrm{E}-02$ & Medium risk \\
\hline Manual start of SBO diesel engine fails & $1.01 \mathrm{E}-02$ & Medium risk \\
\hline Operator fails to switch to CHR system manually & $9.37 \mathrm{E}-03$ & Medium risk \\
\hline Operation possible-fixed-line operation fails-1 & $7.42 \mathrm{E}-03$ & Medium risk \\
\hline Manual isolation of dilution source or corrective action operation fails - EBS is done & $6.31 \mathrm{E}-03$ & Medium risk \\
\hline Manual connection of other ASG-2 water tanks fails & $6.20 \mathrm{E}-03$ & Medium risk \\
\hline Manual connection of other ASG-3 water tanks fails & $6.11 \mathrm{E}-03$ & Medium risk \\
\hline Manual connection of other ASG- 1 water tanks fails & $5.40 \mathrm{E}-03$ & Medium risk \\
\hline
\end{tabular}


stages of NPPs design, as the identification of safety-related human actions is important for NPPs safety. This study first analyzed the importance measures in PSA and, then, the HRAW was proposed based on the RAW; as the basis for a probabilistic method of screening out safetyrelated human actions. The method is suitable for engineering application and provides methodological support to the probabilistic screening method of human actions. As it is traceable and easy to use, it not only can be used in the design of NPPs, but also to provide guidelines in certain aspects for the reviewers to evaluate the NPPs safety.

In addition, there is another significant finding in this study. As shown in Fig. 2, when the CDF is $<1 \mathrm{E}-4$, fewer human actions are listed in the medium-risk range, indicating that the smaller CDF in the NPPs, the higher tolerance of the NPPs to human errors. When the CDF is between $1 \mathrm{E}$ and 4 and $1 \mathrm{E}-5$, it is more likely that human actions be classified as medium risk. When the CDF tends to be $1 \mathrm{E}-4$, it is possible to classify human actions as high risk, even if the HRAW value is small. In this case, the NPP has low tolerance to human errors, and the potential risks are high. Then, it seems that in order to ensure that NPPs have high tolerance to human errors, the CDF should be at least an order of magnitude smaller than the value of the probabilistic safety goal. Based on this finding, it seems that a relationship between CDF and tolerance of human error can be deduced. This will be the subject of future study.

\section{Acknowledgments}

This work was supported by the National Natural Science Foundation of China (Grant No. 71501068, 71771084, 71371070, 51674145), Hunan Provincial Innovation Foundation for Postgraduate (Grant No. CX2017B538), Science and Technology Program of Hunan Province, China (Grant No. 2016WK2006), General Design Assessment (GDA) of the UK HPR1000 Reactor, Research Study and Innovation Experiment Program for University Students of Hunan Province, China (Grant No. Xiangjiaotong [2016]283), and CES-Kingfar Excellent Young Scholar Joint Research Funding. Yanhua Zou was supported by the China Scholarship Council for one year study at Politecnico di Milano.

\section{References}

Apostolakis, G.E., 1989. Uncertainty in probabilistic safety assessment. Nucl. Eng. Design 115 (1), 173-179.

Čepin, M., 2005. Analysis of truncation limit in probabilistic safety assessment. Reliab. Eng. Syst. Safety 87 (3), 395-403.

Čepin, M., 2008. DEPEND-HRA - a method for consideration of dependency in human reliability analysis. Reliab. Eng. Syst. Safety 93 (10), 1452-1460.

Chen, Y., Li, C., Fu, Z., Song, W., Zhang, C., 2013. Study on the Relationship Between PSA and Safety Objectives of Nuclear Power Plant. International Conference on Nuclear Engineering.

China National Nuclear Safety Administration, 2006. Assessment and Validation on the
Power and Safety of. Nuclear Power Plant (HAD102/17).

Dhillon, B.S., 2013. Human Reliability: with Human Factors. Elsevier.

Heng, Zheng, Zhou, Haijing, 2011. Probabilistic Risk Assessment. National Defense Industry Press.

Higgins, J.C., O'Hara, J.M., Lewis, P.M., Persensky, J., Bongarra, J., 2002. Development of a risk screening method for credited operator actions. Human Factors and Power Plants. IEEE Xplore (pp. 8-7-8-13).

Higgins, J.C., O'Hara, J.M., Lewis, P.M., Persensky, J.J., Bongarra, J.P., Cooper, S.E., 2007. Guidance for the review of changes to human action. NUREG-1764. Nuclear Regulatory Commission, US.

International Atomic Energy Agency Applications of probabilistic safety assessment (PSA) for nuclear power plants, 2001 IAEA-TECDOC-1200.

International Atomic Energy Agency, (2012). Safety of Nuclear Power Plants: Design (No. SSR-2/1 Rev.1). IAEA Safety Standards for protecting people and the environment.

Jizhou, Zhu, 2004. Nuclear Reactor Safety Analysis. Xi'an Jiaotong University Press.

Kerch, S.P., Roth, E.M., Sanacktar, S., 1997. Integration of Human Reliability Analysis with Human Factors Engineering Design Implementation Plan. WCAP-14651. Westinghouse Electric Company.

Kim, I.S., Choi, Y., Jeong, K.M., 2017. A new approach to quantify safety benefits of disaster robots. Nucl. Eng. Technol. 49 (7), 1414-1422.

Liu, P., Li, Z., 2014. Human error data collection and comparison with predictions by SPAR-H. Risk Anal. 34 (9), 1706-1719.

Mancuso, A., Compare, M., Salo, A., Zio, E., 2017. Portfolio optimization of safety measures for reducing risks in nuclear systems. Reliab. Eng. System Safety 167.

Ming, Xiaoyang, Yu, Guangwei, Deng, Shiguang, 2017. In: HFE Application in Human System Interface Design of Nuclear Power Plant. Springer, Cham, pp. 361-370.

Nuclear Energy Institute, 2005. 10 CFR 50.69 SSC Categorization Guideline. Nuclear Regulatory Commission, US NEI-00-04.

Office for Nuclear Regulation, (2014). Safety Assessment Principles for Nuclear Facilities (2014 Edition Revision 0).

O'hara, J.M., Higgins, J.C., Fleger, S.A., Pieringer, P.A., 2012. Human factors engineering program review model (REV.3). NUREG-0711, REV.3. US Nuclear Regulatory Commission.

Prasad, M.H., Vinod, G., Rao, V.V.S.S., 2014. Risk management of NPPS using risk monitors. Int. J. Syst. Assurance Eng. Manage. 6 (2), 1-7.

Rebaiaia, M.L., Ait-Kadi, D., Jamshidi, A., Rahimi, S.A., 2015. A combination of reliability calculus and importance measures for probability risk analysis. International Conference on Control, Engineering \& Information Technology, IEEE, pp. 1-6.

Sallak, M., Schon, W., Aguirre, F., 2013. Extended component importance measures considering aleatory and epistemic uncertainties. IEEE Trans. Reliab. 62 (1), 49-65.

Schulz, T.L., Brockhoff, C.S., Hayes, T.P., 2006. AP1000 Identification of Critical Human Actions and Risk Important Tasks. WCAP-16555. Westinghouse Electric Company.

Siu, N., Stutzke, M., Dennis, S., Harrison, D., 2016. Probabilistic Risk Assessment and Regulatory Decisionmaking: Some Frequently Asked Questions. United States Nuclear Regulatory Commission, Office of Nuclear Regulatory Research.

True, D., Fleming, K., Parry, G., Putney, G., Sursock, J.-P., 1995. PSA Applications Guide. Electric Power Research Institute.

US Nuclear Regulatory Commission, 2011. An Approach for Using Probabilistic Risk-assessment in Risk-informed Decisions on Plant-specific Changes to the Licensing Basis. US Nuclear Regulatory Commission, Office of Nuclear Regulatory Research.

Van der Borst, M., Schoonakker, H., 2001. An overview of PSA importance measures. Reliab. Eng. Syst. Safety 72 (3), 241-245.

Vesely, W.E., Belhadj, M., Rezos, J.T., 1994. PRA importance measures for maintenance prioritization applications. Reliab. Eng. Syst. Safety 43 (3), 307-318.

Zio, E., 2007. An Introduction to the Basics of Reliability and Risk Analysis. World Scientific.

Zio, E., Podofillini, L., 2003. Importance measures of multi-state components in multistate systems. Int. J. Reliab., Quality Safety Eng. 10 (03), 289-310.

Zou, Y., Zhang, L., Dai, L., et al., 2017. Human reliability analysis for digitized nuclear power plants: case study on the LingAo II nuclear power plant. Nucl. Eng. Technol. 49 (2), 335-341. 